OPEN ACCESS

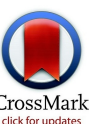

${ }^{1}$ Department of Emergency

Medicine, University of

California, 505 Parnassus Ave,

San Francisco, CA 94143, USA

${ }^{2}$ School of Public Health,

University of California, 239

University Hall \#7360,

University of California,

Berkeley, CA 94720-7360, USA

${ }^{3}$ Department of Emergency

Medicine, University of

California, 1001 Potrero Ave,

San Francisco CA 94143, USA

Correspondence to: J C C Montoy juancarlos.montoy@ucsf.edu

Additional material is published online only. To view please visit the journal online (http://dx.doi. org/10.1136/bmj.h6895)

Cite this as: BMJ 2016;352:h6895 http://dx.doi.org/10.1136/bmj.h6895

Accepted: 25 November 2015

\section{Patient choice in opt-in, active choice, and opt-out HIV screening: randomized clinical trial}

\author{
Juan Carlos C Montoy, ${ }^{1}$ William H Dow, ${ }^{2}$ Beth C Kaplan³
}

\begin{abstract}
STUDY QUESTION

What is the effect of default test offers-opt-in, opt-out, and active choice-on the likelihood of acceptance of an HIV test among patients receiving care in an emergency department?

METHODS

This was a randomized clinical trial conducted in the emergency department of an urban teaching hospital and regional trauma center. Patients aged 13-64 years were randomized to opt-in, opt-out, and active choice HIV test offers. The primary outcome was HIV test acceptance percentage. The Denver Risk Score was used to categorize patients as being at low, intermediate, or high risk of HIV infection.
\end{abstract}

\section{STUDY ANSWER AND LIMITATIONS}

$38.0 \%(611 / 1607)$ of patients in the opt-in testing group accepted an HIV test, compared with $51.3 \%$ $(815 / 1628)$ in the active choice arm (difference 13.3\%, $95 \%$ confidence interval $9.8 \%$ to $16.7 \%$ ) and $65.9 \%$ $(1031 / 1565)$ in the opt-out arm (difference $27.9 \%$, $24.4 \%$ to $31.3 \%$ ). Compared with active choice testing, opt-out testing led to a 14.6 (11.1 to 18.1) percentage point increase in test acceptance. Patients identified as being at intermediate and high risk were more likely to accept testing than were those at low risk in all arms (difference $6.4 \%$ (3.4\% to $9.3 \%$ ) for intermediate and $8.3 \%$ (3.3\% to $13.4 \%$ ) for high risk). The opt-out effect was significantly smaller among those reporting high risk behaviors, but the active choice effect did not significantly vary by level of reported risk behavior. Patients consented to inclusion in the study after being offered an HIV test, and inclusion varied slightly by treatment assignment. The study took place at a single county hospital in a city that is somewhat unique with respect to HIV testing; although the test acceptance percentages themselves might vary,

\section{WHAT IS ALREADY KNOWN ON THIS TOPIC}

Patients' preferences are a hallmark of patient centered care, but little is known about how wording of offers of testing can influence perceived preferences

Opt-in and opt-out HIV testing have not been compared in a randomized controlled setting

US guidelines endorse opt-out HIV testing, and Europe has seen a trend toward this testing scheme

\section{WHAT THIS STUDY ADDS}

Opt-in and opt-out defaults had statistically and clinically significant effects on the likelihood of patients accepting tests

Patients reporting risk factors were more likely to accept testing in each testing regimen than were patients reporting no risk factors

Active choice is a distinct test regimen, with test acceptance patterns that may best approximate patients' true preferences versus opt-out test schemes would not be expected.

WHAT THIS PAPER ADDS

Active choice is a distinct test regimen, with test acceptance patterns that may best approximate patients' true preferences. Opt-out regimens can substantially increase HIV testing, and opt-in schemes may reduce testing, compared with active choice testing. FUNDING, COMPETING INTERESTS, DATA SHARING This study was supported by grant NIA 1RC4AG039078 from the National Institute on Aging. The full dataset is available from the corresponding author. Consent for data sharing was not obtained, but the data are anonymized and risk of identification is low.

TRIAL REGISTRATION

Clinical trials NCT01377857.

\section{Introduction}

Opt-out HIV testing has received a great deal of attention since the United States Centers for Disease Control and Prevention (CDC) revised its HIV testing guidelines in 2006 to recommend non-targeted opt-out testing. . $^{1-3}$ The CDC noted that emergency departments are especially well situated to identify the estimated $20 \%$ of HIV positive people who do not have a diagnosis. ${ }^{4-7}$ Governments and health departments elsewhere have likewise made a push toward opt-out testing to identify HIV infected people earlier in the course of their disease, although the specifics of whom to test and in what setting remain highly regionalized. ${ }^{8-12}$ However, most hospitals have not acted on the CDC's opt-out recommendations, in part because of unanswered questions about the diagnostic yield of such testing. ${ }^{1314}$

The effect of opt-in versus opt-out defaults has been identified in other settings, ${ }^{1516}$ but it has not been as carefully identified for HIV testing; the CDC's endorsement for opt-out testing was based on thin evidence of its efficacy in increasing patients' acceptance of HIV screening. ${ }^{17-19}$ Subsequent studies of emergency department based HIV testing have shown that opt-out testing programs can be successfully implemented and are associated with testing of a higher proportion of patients, but they report highly varied test acceptance percentages ranging from $29 \%$ to $87 \% .^{20-29}$ The wide range in reported test acceptance percentages suggests that the details of the testing regimen-including how the test is offered, by whom, to whom, and in what setting-can be crucial to how likely patients are to agree to be tested..$^{30}$ For example, two conflicting precursor studies from a single institution found significantly higher and significantly lower test acceptance percentages for opt-out compared with opt-in testing, suggesting that other changes in the method of offering the test may a different pattern for opt-in versus active choice 
have outweighed any effect of moving from opt-in to opt-out testing. ${ }^{2031}$

The study presented in this paper systematically isolated the effect of test defaults by randomizing patients to opt-in versus opt-out offers while holding all else constant. We hypothesized that some of the variation in results previously seen for opt-out testing schemes stems from heterogeneity across studies in the comparison opt-in regimen, as well as the precise nature of the opt-out regimen implemented. We also wanted to answer various research questions identified by consensus research recommendations that noted the significant knowledge gap regarding opt-in and opt-out testing and thus recommended prioritizing the study of factors influencing patients' acceptance of HIV testing. ${ }^{3233}$ In addition, we estimated risk of infection by using self reported behaviors collected from a questionnaire that we administered as part of the study.

We also defined a default-free "active choice" regimen in which patients were asked to choose whether they would like to be tested or not. We distinguish this from an opt-in program in which patients are informed that tests are available but not tested unless they request to be tested. Although this distinction may be subtle, we hypothesized that it is useful because it could result in clinically significant differences in behavior and help to identify a mechanism that influences perceived patient preferences.

We examined active choice and opt-out HIV testing schemes in comparison with opt-in. We also controlled for all other aspects of the test offer, including informing patients in all default assignments that testing is available so as to specifically isolate the effects of defaults as distinct from information or other confounding features. We used self reported risk factors to test whether opt-out testing disproportionately increased testing among people at lower rather than higher risk. We hypothesized that a greater proportion of patients would be tested under an active choice scheme than under an opt-in scheme, and that a greater proportion would test under the opt-out scheme than the active choice scheme. We also hypothesized that these differences would be observed within each risk group.

\section{Methods}

We did a non-blinded, randomized clinical trial in the emergency department of an urban teaching hospital and regional trauma center. Between 18 June 2011 and 30 June 2013, non-clinical staff approached patients during their visit to the emergency department: once to offer them a questionnaire and once to offer them a rapid HIV test. Study staff approached patients in parallel with their standard care. The test offers were made as if they were a component of patients' care in the department. Questionnaires were presented with a generic description of a 10 minute questionnaire about improving emergency department care. After the second of these visits, patients were made aware that the test offer and questionnaire were part of a study; they were fully debriefed and gave consent. The study was conducted and reported in accordance with CONSORT guidelines.
Opt-in, active choice, and opt-out treatment assignments were randomized at the level of the patient; we used a random number generator to create individual assignments with equal probability. Patients were also randomly assigned to be offered the questionnaire either before or after the offer of an HIV test (1:1 allocation); the treatment assignments were cross randomized in a factorial design. Study staff had sheets with unconcealed treatment assignments to which they sequentially assigned participants. In results reported elsewhere, another sample of patients was randomized to receive small monetary incentives for testing, but for clarity of interpretation we report here results only for participants who were offered no monetary incentives. These patients were not aware of the monetary incentives offered to other patients.

No incentive was offered for completion of the questionnaire, which was self administered using iPads. It elicited demographic information, HIV related risk behaviors (such as number of sex partners, condom use, and drug use), and beliefs about HIV infection and its consequences (such as the estimated life expectancy for people infected with HIV), as well as eliciting patients' subjective assessment of their risk of infection.

Study staff worked two or three non-overlapping five hour shifts a day for a total of 20 shifts a week. They started each shift in one of four emergency department zones according to a calendar created using a random number generator to assign starting zones with equal probability. After exhausting all eligible patients in a given zone, staff moved to the next zone.

\section{Participants}

Patients were eligible for inclusion in the study if they were between 13 and 64 years old, were able to consent to HIV testing and to study inclusion, and spoke either English or Spanish. We excluded patients if they had previously been diagnosed as having HIV infection, had received an HIV test in the previous three months, were pregnant, were in police custody, or had participated in this study in the previous three months. For patients presenting with altered mental status, study staff determined their ability to consent with the nurse or clinician at the time of approach.

\section{Patient involvement}

No patients were involved in setting the research question or the outcome measures, nor were they involved in recruitment, or the design and implementation of the study. There are no plans to involve patients in dissemination.

\section{Protocol}

Study staff identified potential patients by using the electronic medical record. They approached patients to offer either an HIV test or the questionnaire, according to their treatment assignment. All participants were informed that the emergency department was offering rapid screening HIV tests in a non-targeted manner to all patients. Scripts standardized the provision of information about the availability of HIV testing and test 
offers: "We're offering routine HIV tests to all of our patients. It's a rapid test with results available in one to two hours." The test offer followed-opt-in: "You can let me, your nurse, or your doctor know if you'd like a test today;” active choice: "Would you like a test today?” or opt-out: "You will be tested unless you decline."

Study staff offered tests and obtained verbal acceptance; they notified clinicians of patients accepting HIV tests. No pre-test counseling was offered. Test results were available and reported to patients after completion of the treatments and debriefing. Patients were informed of negative test results by their nurse or clinician. Positive test results were disclosed by the patient's clinician in accordance with the protocol established by the hospital's HIV rapid testing and referral program.

Patients were not made aware of the study itself until after both the test and questionnaire offers, at which time they were fully debriefed and asked to consent to inclusion in the study. No incentive was offered for study participation. All approached patients were asked to consent regardless of whether they agreed to the test or completed the questionnaire.

\section{Statistics}

We used a $\chi^{2}$ test to assess allocation of patients to treatment assignments according to observable characteristics; we used a test of trend for ordinal categories. Although patients were randomized, the design included a retrospective debrief and consent, and treatment allocation was not concealed, so some risk of biased treatment assignments existed.

The primary outcome was test acceptance percentage. In addition to overall acceptance and acceptance percentage by treatment, we examined test acceptance for subgroups defined by HIV related risk behaviors, adjusted for demographics; we estimated treatment effects from univariate and multivariate ordinary least squares regressions. In the tables, we report raw linear regression coefficients, which have the benefit that they are directly interpretable as the difference in the proportion of participants who accepted an HIV test; interaction effects between study arm and risk level are similarly easily interpretable. ${ }^{34}$ Because the test acceptance rate is in the middle part of the distribution, results would be quite similar if we used a non-linear model such as logistic regression instead. We estimated risk of infection according to our estimates of their Denver HIV Risk Score. ${ }^{35} 36$ Points are given for age, sex, race/ethnicity, sex with a male partner, vaginal intercourse, receptive anal intercourse, intravenous drug use, and past HIV testing (appendix table A). We classified patients with a score less than 20 as being at low risk, those scoring 20-39 as at intermediate risk, and those scoring 40 or higher as at high risk. For patients who did not complete the questionnaire, we estimated the risk score by using available data. Analysis by risk level was a planned analysis, but these risk definitions were not pre-specified, as the Denver HIV Risk Score was published and validated during our data collection. All standard errors are clustered by day and emergency department zone (day-zone level). Sensitivity analyses including additional covariates, different risk specifications, and multivariate logistic regression are presented in the appendix. We used Stata 13.1 for randomization and all analyses.

The study's originally planned sample size was sufficient to detect a 5 percentage point difference in test acceptance percentage between treatment arms with $93 \%$ power at a $5 \%$ significance level. This 5 percentage point effect size was the minimum difference we deemed to be clinically important. We assumed a baseline test acceptance percentage of $50 \%$. The power of $93 \%$ resulted from sample size needs for the larger study of which this is a part. The sample size needed to achieve a minimum of $80 \%$ power for the main comparisons across arms in the analysis presented here was 1565 patients per group. Our actual enrolled sample size was smaller than originally planned owing to enrollment difficulties, but it met this 1565 minimum; thus the sample was sufficient for achieving $80 \%$ power for the main comparisons across arms presented in the analyses below.

\section{Results}

Of 5801 patients approached by study staff, 4800 (82.7\%) consented to study inclusion: 1607 (82.7\%) in the opt-in group, $1628(81.0 \%)$ in the active choice group, and 1565 (84.7\%) of the opt-out group $\left(\chi^{2} \mathrm{P}<0.01\right)$. Of those who consented to inclusion, $33.5 \%$ were randomized to opt-in, $33.9 \%$ to active choice, and $32.6 \%$ to opt-out test offers. Figure 1 shows the flow of patients

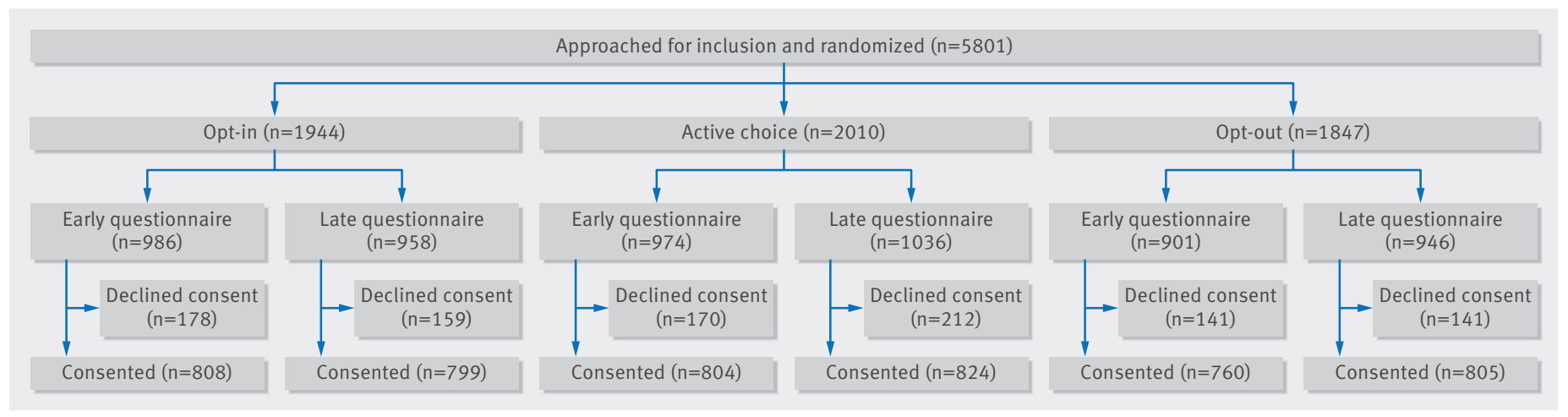

Fig 1 Flow chart of HIV Defaults Study. Of 5801 patients approached for inclusion in study, 4800 consented. Of those approached, $33.5 \%, 34.7 \%$, and $31.8 \%$ were assigned to opt-in, active choice, and opt-out test treatments, respectively. The final study population comprised $33.5 \%$ opt-in, $33.9 \%$ active-choice, and $32.6 \%$ opt-out patients 


\begin{tabular}{|c|c|c|c|c|c|}
\hline Characteristic & All $(n=4800)$ & Opt-in $(\mathrm{n}=1607)$ & Active choice $(n=1628)$ & Opt-out $(n=1565)$ & Pvalue* \\
\hline \multicolumn{6}{|l|}{ Demographics } \\
\hline Male sex & $2887(60.1)$ & $992(61.7)$ & $991(60.9)$ & $904(57.8)$ & 0.057 \\
\hline Median (interquartile range) age, years, & $42(31-53)$ & $42(30-53)$ & $42(31-53)$ & $43(31-53)$ & 0.411 \\
\hline \multicolumn{6}{|l|}{ Racet: } \\
\hline American Indian/Alaska Native & $59(1.2)$ & $17(1.1)$ & $15(0.9)$ & $27(1.7)$ & \multirow{6}{*}{0.032} \\
\hline Asian & $451(9.4)$ & $134(8.3)$ & $159(9.8)$ & $158(10.1)$ & \\
\hline Black & $1248(26.0)$ & $432(26.9)$ & $404(24.8)$ & $412(26.3)$ & \\
\hline Native Hawaiian/Pacific Islander & $140(2.9)$ & $46(2.9)$ & $42(2.6)$ & $52(3.3)$ & \\
\hline White & $2676(55.8)$ & $914(56.9)$ & $914(56.1)$ & $848(54.2)$ & \\
\hline Unknown & $330(6.9)$ & $91(5.7)$ & $135(8.3)$ & $104(6.7)$ & \\
\hline Latino ethnicity & $1163(24.2)$ & $394(24.5)$ & $370(22.7)$ & $399(25.5)$ & 0.179 \\
\hline Education $\geq$ high school & $2844(59.3)$ & $941(58.6)$ & $964(59.2)$ & $939(60.0)$ & 0.710 \\
\hline Identifies as lesbian, gay, or bisexual & $589(12.3)$ & $206(12.8)$ & $188(11.5)$ & $195(12.5)$ & 0.524 \\
\hline \multicolumn{6}{|l|}{ Chief complaint } \\
\hline Abdominal /gastrointestinal & $979(20.4)$ & $396(24.6)$ & $338(20.8)$ & $345(22.0)$ & \multirow{15}{*}{0.043} \\
\hline Cardiovascular & $544(11.3)$ & $183(11.4)$ & $167(10.3)$ & $194(12.4)$ & \\
\hline Endocrine & $61(1.3)$ & $21(1.3)$ & $21(1.3)$ & $19(1.2)$ & \\
\hline General & $288(6.0)$ & $88(5.5)$ & $98(6.0)$ & $102(6.5)$ & \\
\hline Genitourinary/renal & $302(6.3)$ & $106(6.6)$ & $91(5.6)$ & $105(6.7)$ & \\
\hline Musculoskeletal & $763(15.9)$ & $282(17.5)$ & $250(15.4)$ & $231(14.8)$ & \\
\hline Stroke & $18(0.4)$ & $5(0.3)$ & $9(0.6)$ & $4(0.3)$ & \\
\hline Neurological (non-stroke) & $296(6.2)$ & $112(7.0)$ & $99(6.1)$ & $85(5.4)$ & \\
\hline Oral/dental & $69(1.4)$ & $27(1.7)$ & $19(1.2)$ & $23(1.5)$ & \\
\hline Psychiatric & $52(1.1)$ & $15(0.9)$ & $17(1.0)$ & $20(1.3)$ & \\
\hline Respiratory & $372(7.8)$ & $120(7.5)$ & $131(8.0)$ & $121(7.7)$ & \\
\hline Skin & $386(8.0)$ & $143(8.9)$ & $141(8.7)$ & $102(6.5)$ & \\
\hline Substance intoxication/withdrawal & $92(1.9)$ & $25(1.6)$ & $40(2.5)$ & $27(1.7)$ & \\
\hline Trauma & $426(8.9)$ & $143(8.9)$ & $139(8.5)$ & $140(8.9)$ & \\
\hline Other & $156(3.3)$ & $41(2.6)$ & $68(4.2)$ & $47(3.0)$ & \\
\hline \multicolumn{6}{|l|}{ Risk of infection } \\
\hline Low risk & $1943(40.5)$ & $618(38.5)$ & $689(42.3)$ & $636(40.6)$ & \multirow{3}{*}{0.387} \\
\hline Medium risk & $2388(49.8)$ & $830(51.7)$ & $788(48.4)$ & $770(49.2)$ & \\
\hline High risk & $469(9.8)$ & $159(9.9)$ & $151(9.3)$ & $159(10.2)$ & \\
\hline \multicolumn{6}{|l|}{ HIV test history } \\
\hline Ever tested & $3880(80.8)$ & 1309 (81.5) & $1302(80.0)$ & $1269(81.1)$ & 0.538 \\
\hline Tested in past 6 months & $910(19.0)$ & $290(18.0)$ & $311(19.1)$ & 309 (19.7) & 0.467 \\
\hline Unreported test history & $971(20.2)$ & $341(21.2)$ & $334(20.5)$ & $296(18.9)$ & 0.254 \\
\hline Refused questionnaire & $940(19.6)$ & $329(20.5)$ & $321(19.7)$ & $290(18.5)$ & 0.336 \\
\hline
\end{tabular}

through the study, and table 1 shows their demographics and chief presenting complaint. Race and chief complaint were not equally distributed across treatment assignments ( $\chi^{2} \mathrm{P}=0.032$ and 0.043 , respectively); no tests of independence between the three treatment assignments were significant at the $\mathrm{P}<0.05$ level after Bonferroni correction (P values in table 1 have not been Bonferroni corrected).

\section{HIV test acceptance percentage}

Patients accepted $51.6 \%$ of all offers of tests. Opt-in, active choice, and opt-out test offers resulted in test acceptance percentages of $38.0 \%, 51.3 \%$, and $65.9 \%$, respectively (fig 2 , all patients). The unadjusted differences reflect an absolute difference in HIV testing percentage across defaults of $13.3 \%$ ( $95 \%$ confidence interval $9.8 \%$ to $16.7 \%$ ) in the active choice arm compared with opt-in and an absolute difference of $27.9 \%$

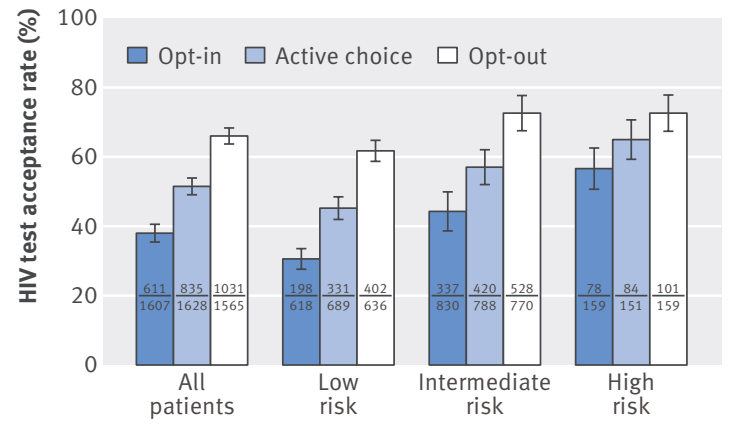

Fig 2 | HIV test acceptance percentage by risk of infection: unadjusted results. Test acceptance percentage is shown according to treatment assignment (opt-in, active choice, and opt-out), and according to risk of HIV infection. Lines indicate $95 \%$ confidence intervals. Numbers of patients from each risk category accepting and offered HIV testing under each treatment group are presented as numerator and denominator 


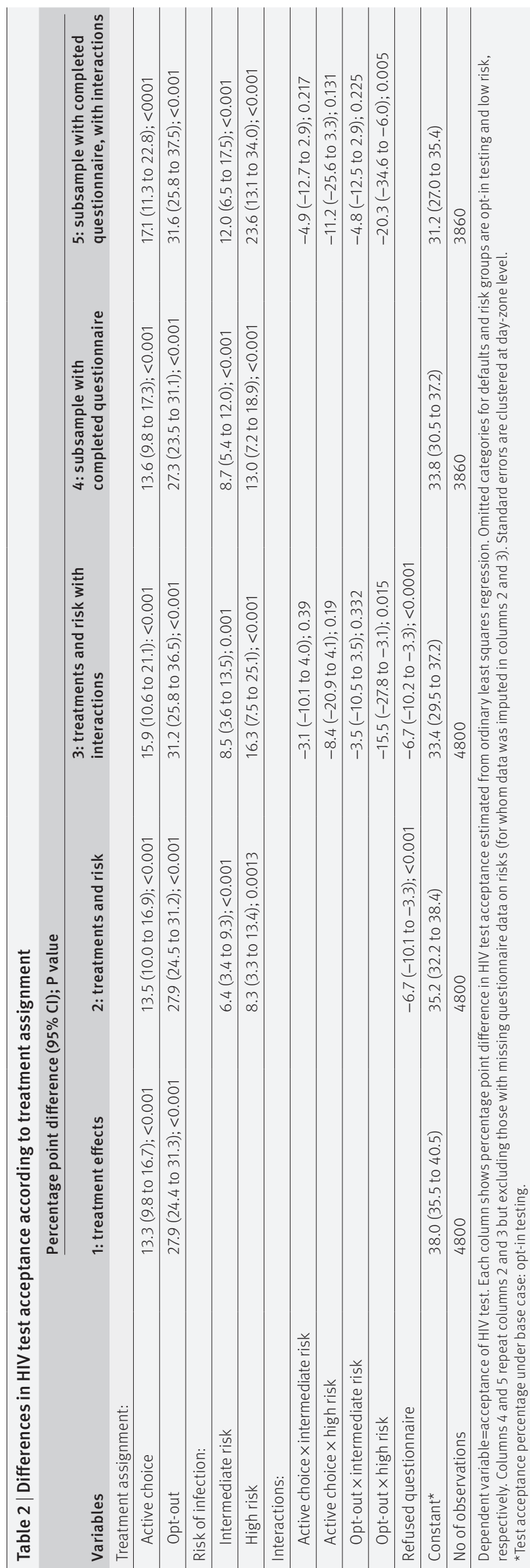

(24.4\% to $31.3 \%$ ) in the opt-out arm compared with opt-in (table 2, column 1). Compared with active choice testing, opt-out testing yielded $14.6 \%$ (11.1\% to $18.1 \%)$ more test acceptances. Figure 2 illustrates unadjusted test acceptance percentages by treatment assignment for each of the risk groups.

Table 2 shows results from multivariate ordinary least squares regressions of HIV test acceptance percentages according to treatment assignment. Test acceptance percentages were higher for patients with higher Denver HIV Risk Scores (table 2, column 2; also appendix table $\mathrm{C}$ ). When risk specific interactions with treatment assignments were included in the regression (table 2, column 3 ) the active choice and opt-out effects were larger for the (comparison) low risk group. We did not find risk specific differences in treatment effects for patients at intermediate risk. For patients at high risk, the effect of active choice was not significantly modified but the effect of opt-out was attenuated (interaction term between opt-out and high risk: $-15.5 \%, 95 \%$ confidence interval -27.8 to -3.1 ). Patients who did not complete the questionnaire had incompletely measured Denver HIV Risk Scores. Columns 4 and 5 of table 2 show the results of the same models as in columns 2 and 3, respectively, with the sample restricted to those who completed the questionnaire; results differ only slightly. In this subsample, a larger difference existed in testing proportions according to risk level. Again, we did not find risk specific differences in treatment effects except for high risk patients in the opt-out treatment arm.

Results did not change after adjustment for chief complaint (data not shown). Patients offered the questionnaire first were less likely to accept a test than were those offered the test first; however, we found no significant interaction between the timing of the questionnaire and treatment assignment with respect to test acceptance percentage (appendix figure A). Likewise, multivariate ordinary least squares and logistic regressions showed that the differences in test acceptance by treatment assignment were unchanged after we controlled for risk of infection, demographics, and HIV test history (appendix tables D and E). Patients who had never been tested for HIV accepted 48.8\% (449/920) of test offers; $52.3 \%$ (2028/3880) of patients who had previously been tested accepted the test. Alternative risk classifications and sensitivity analyses are shown in the appendix.

Effects of test offer scripts persisted when dummy variable fixed effect indicator variables for each study staff member who offered tests were included in the specification. Figure 3 shows test acceptance according to research staff member. Staff (a) is a composite of staff members who saw fewer than 200 patients each; staff members (b) to (i) each consented 200 or more patients. Test acceptance trended higher in the active choice than the opt-in treatment arm for each staff member; it also trended higher in the opt-out than active choice treatment arm, although substantial heterogeneity exists in the magnitude of default effects across the staff. 


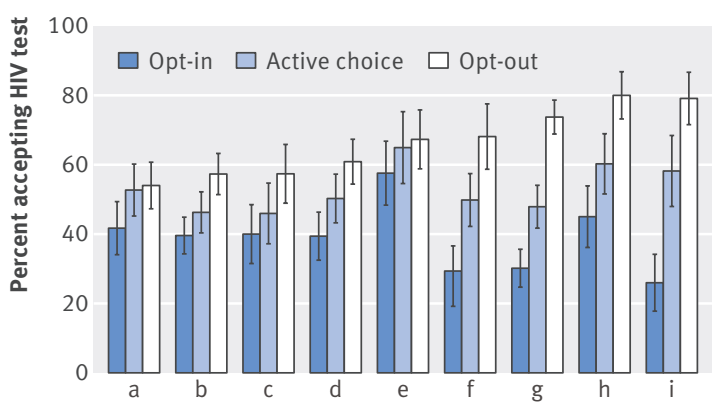

Fig 3 | Test acceptance percentage by study staff member; (a) is composite of study staff who saw fewer than 200 patients each; (b) to (i) are individual staff members who saw more than 200 patients each. Lines indicate $95 \%$ confidence intervals

Numbers of patients agreeing to and offered testing according to treatment assignment and staff member are shown in appendix table $\mathrm{F}$.

\section{Discussion}

Our study provides evidence that small changes in wording can significantly affect patients' behavior and thus our understanding of their preferences. Specifically, modifying HIV testing defaults led to clinically and statistically significant differences in test acceptance percentages. Holding all else constant (including notifying all patients that HIV testing was available), opt-out test offers were accepted 28 percentage points more often than opt-in offers and 13 percentage points more often than active choice offers. We found that active choice testing, although previously considered a form of opt-in testing, is a distinct category: compared with a strict opt-in scheme informing patients that they can request a test, simply asking patients if they would like a test increased test acceptance by 13 percentage points.

\section{Strengths and weaknesses of study}

Patients with a wide range of demographics, chief complaints, and reported risk factors for HIV were randomized at the patient level to a one sentence variation in test offer, with all else held constant. Thus, we were able to identify the effect of opt-in and optout defaults compared with requiring patients to state their testing preference (active choice). Although the finding that opt-out testing yielded the highest test acceptance percentage is unsurprising, the greater precision with which this effect was measured is novel, as is its measurement across populations reporting different risks. This study took place at a single county hospital in a city that is somewhat unique with respect to HIV testing, and the proportion of patients accepting testing may vary in other settings. However, although the test acceptance percentages themselves might vary, we have little reason to expect a different pattern for opt-in versus active choice versus opt-out test schemes. Likewise, although the particular percentages may be quite different, this patterned response to op-in, active choice, and opt-out test offers may be expected for decisions about other medical tests.

Using non-clinical staff had the advantage of standardizing all aspects of the test offer, although this somewhat limits the generalizability of the results. The patterns across treatment arms were evident for each of the study staff who approached patients, and we expect that they would persist outside the context of this study. Likewise, the variation in test acceptance by offerer would also be expected to be present outside of this study. This variation may help to explain the highly variable opt-out results found across previous studies, particularly when the precise wording of the test was not standardized or reported. No default regimen approached 100\% acceptance for any treatment or study staff member, even when the data were examined according to risk level.

Our study did not test a randomized "usual care" arm in which opt-in participants were not routinely informed of the availability of HIV testing. For reference, in a comparable set of patients receiving usual care in the three months after study conclusion, only $3 \%$ of patients were tested for HIV; this comprises patients tested diagnostically and patients who initiated the test by their own request. The opt-in test acceptance percentage was substantially higher than the post-study HIV test percentage, suggesting that simply mentioning the availability of HIV testing to every patient could non-trivially increase HIV testing and that some of the increase in test acceptance seen in previous studies was due to increased information provided to patients rather than the opt-out default. This study included a questionnaire, which is not likely to be standard practice in emergency departments, although it has been implemented in other studies of HIV testing and may become more commonplace as brief interventions take hold on a larger scale. ${ }^{37-39}$ We found no difference in treatment effects between patients who declined to complete the questionnaire and those who completed the questionnaire.

Retrospective informed consent has the advantage of minimizing many potential sources of bias but runs the risk of introducing bias from post-randomization withdrawal. However, given the similar participation rates across treatment arms and successful randomization across many observables, this is quite unlikely to have driven our results. Study staff were not blinded to treatment assignments before assigning them to patients. They were instructed to enroll patients sequentially according to availability within each zone, and this was reinforced by study team meetings and oversight from the research coordinator. We cannot exclude the possibility that staff occasionally violated protocol and approached patients in a biased manner. However, given our consistent results across research staff (fig 3) and successful randomization with similar baseline characteristics across arms (table 1), we feel that it is quite unlikely that study staff selected patients or that patients refused study participation in a manner that biased our results. 


\section{Strengths and weaknesses in relation to other studies}

To our knowledge, this is the first study of HIV testing to estimate explicitly the effects of defaults as distinct from information and to test the effects of an active choice regimen. Previous work has provided less explicit findings on the precise nature of the default regimen, as studies were often performed on before and after policy changes with schemes that modified other aspects of the testing scheme as well. In addition to comparing opt-in and opt-out testing schemes, we defined and measured test acceptance under a new category, active choice testing, which had previously been considered a form of opt-in testing. The distinction between our opt-in and active choice arms is subtle, but the clinically significant difference in the proportion agreeing to a test between them suggests that minor policy variations can have large effects. Active choice testing balances the goals of increasing HIV screening and fostering patient centered decision making. The active choice option, however, raised HIV testing percentages only half as much as did the opt-out regimenan important trade-off to consider.

We found that patients self identifying as at risk for HIV infection were more likely to test in each treatment scheme than were patients reporting no risks. Identifying risk from self reported behavior is not without limitations, but previous work has tended either not to collect this information or to use it to assess the utility of targeted testing, rather than to consider how policy changes would affect test acceptance among patients of various risk levels. ${ }^{1425}$ Future work is needed to incorporate our findings into cost effectiveness models of optimal testing strategies.

\section{Meaning of study}

The variation in test acceptance percentages according to test offer scheme shows that measuring patients' preferences is not necessarily straightforward. A central tenet of patient centered care is the idea that patients' preferences should be factored into healthcare decisions. However, if small changes in the way we ask patients about their preferences significantly affect their answers, accurately identifying patients' true preferences may not be as simple as one might expect.

Previous research has shown that test acceptance is not solely related to perceived risk but can be affected by non-risk related factors as well. ${ }^{40-42}$ The finding that even patients reporting multiple risk factors for HIV declined testing suggests that avoidance of information may play a role in the decision whether to test and raises concern that universal offers of testing may not identify all people with undiagnosed HIV infection. Our instrument did not use identical questions to the Denver HIV Risk Score instrument, but these findings were robust to alternative specifications of risk, and the possible differences in risk score measurement are unlikely to change the interpretation of our results.

The pattern of test acceptance is consistent with the hypothesis that some patients accept defaults regardless of their actual testing preferences. Possible explanations of the substantial effects induced by minor variations in wording include passive decision making, the effort involved in speaking up, inattention, and concern about rejecting medical advice (particularly during an episode of emergency care). ${ }^{43-48}$ Fear of being stigmatized on the basis of a request for or acceptance of an HIV test, small immediate costs outweighing large but distant benefits, and the desire to preserve hope rather than learn bad news may partially explain why patients decline testing, but it would be surprising for stigma, myopic decision making, or emotional self regulation to explain the differential responses between the three treatments. ${ }^{49-51}$ Likewise, length of emergency department visit and pain may influence testing decisions, but why these factors should affect responses differentially by type of test offer is not clear.

The US National Emergency Department HIV Testing Consortium defines opt-in testing as tests "presented so the patient would be expected to understand the default is to not test unless he or she states agreement." 52 We propose the following clarifications to the definition of opt-in testing: refine opt-in to describe a default of no test unless one is affirmatively requested. Just as opt-out testing describes a scheme in which patients can explicitly decline or implicitly accept a test, opt-in testing should describe a symmetric scheme in which patients can explicitly accept or implicitly decline a test. We also propose the conceptual establishment of a third regimen, active choice testing, in which patients are prompted to state their testing preference, thereby effectively removing any default. Simply asking patients, "Would you like a test?" encourages them to consider options proactively, consistent with the movement toward enhanced patient decision making.

We tested the hypothesis that moving from opt-in to opt-out regimens could disproportionately induce testing among people at low risk, thus wasting resources; our results only partially support this hypothesis. Test acceptance percentages increased strongly and significantly among all risk groups when moving from opt-in to opt-out. The effect of moving from opt-in to opt-out was somewhat smaller in the high risk group, but test acceptance was more likely in all arms among those patients with higher self reported risk factors, and the smaller opt-out effect may simply be a mechanical effect of already high test acceptance percentages in the population tested. Furthermore, the difference in acceptance between opt-in and active choice testing did not significantly vary across risk groups.

\section{Future research}

Understanding how defaults shape behavior for patients with different preferences is crucial to providing patient centered care. Careful use of defaults has the potential to improve patients' health across many domains, particularly for preventive health or behaviors with immediate costs and delayed benefits such as smoking cessation or colonoscopy. There is a balance between preserving patients' autonomy and steering them toward care that optimizes health. This is of particular importance when patients may incorrectly 
perceive themselves to be at low risk or when those at greatest risk may be more likely to decline testing. Furthermore, as the field continues to advance patient centered decision making, and as the Affordable Care Act ties reimbursement to engagement of patients, defaults should be given particular consideration. Although decision aids have been shown to influence patients' decisions, this study shows that a one sentence variation can dramatically affect their decisions and, likewise, our perceptions of their preferences. ${ }^{53}$

Contributors: All authors were involved in the study concept and design and in obtaining funding. JCCM and WHD were involved in the acquisition, analysis, and interpretation of data. JCCM drafted the manuscript, and all authors critically revised it for important intellectual content. JCCM and WHD did the statistical analysis. BCK provided administrative, technical, and material support. JCCM is the guarantor.

Funding: This study was supported by grant NIA 1RC4AG039078 from the National Institute on Aging (awarded to WHD and BCK). The content is solely the responsibility of the authors and does not necessarily represent the official views of the National Institute on Aging or the National Institutes of Health. The sponsors had no role in the design and conduct of the study; collection, management, analysis, and interpretation of the data; preparation, review, or approval of the manuscript; or the decision to submit the manuscript for publication.

Competing interests: All authors have completed the ICMJE uniform disclosure form at www.icmje.org/coi_disclosure.pdf and declare: support from the National Institute on Aging for the submitted work; no financial relationships with any organizations that might have an interest in the submitted work in the previous three years; no other relationships or activities that could appear to have influenced the submitted work.

Ethical approval: The study received institutional review board approval from the University of California, San Francisco.

Data sharing: The full dataset is available from the corresponding author at juancarlos.montoy@ucsf.edu. Participants gave informed consent for data collection; consent for sharing was not obtained, but the presented data are anonymized and risk of identification is low.

Transparency statement: The lead author (the manuscript's guarantor) affirms that the manuscript is an honest, accurate, and transparent account of the study being reported; that no important aspects of the study have been omitted; and that any discrepancies from the study as planned (and, if relevant, registered) have been explained.

This is an Open Access article distributed in accordance with the Creative Commons Attribution Non Commercial (CC BY-NC 3.0) license, which permits others to distribute, remix, adapt, build upon this work non-commercially, and license their derivative works on different terms, provided the original work is properly cited and the use is non-commercial. See: http://creativecommons.org/licenses/ by-nc/3.0/.

1 Branson BM, Handsfield HH, Lampe MA et al. Centers for Disease Control and Prevention (CDC). Revised recommendations for HIV testing of adults, adolescents, and pregnant women in health-care settings. MMWR Recomm Rep 2006;55(RR-14): 1-17, quiz CE1-4. 16988643

2 Brown J, Shesser R, Simon G et al. Routine HIV screening in the emergency department using the new US Centers for Disease Control and Prevention Guidelines: results from a high-prevalence area. | Acquir Immune Defic Syndr 2007;46: 395-401. doi:10.1097| QAl.0b013e3181582d82 18077831

3 Merchant RC, Seage GR, Mayer KH, Clark MA, DeGruttola VG, Becker BM. Emergency department patient acceptance of opt-in, universal, rapid HIV screening. Public Health Rep 2008;123(Suppl 3): 27-40. 19172704

4 Qaseem A, Snow V, Shekelle P, Hopkins R Jr, Owens DK. Clinical Efficacy Assessment Subcommittee, American College of Physicians. Screening for HIV in health care settings: a guidance statement from the American College of Physicians and HIV Medicine Association. Ann Intern Med 2009;150: 125-31. doi:10.7326/0003-4819-150-2200901200-00300 19047022

5 Centers for Disease Control and Prevention (CDC). HIV surveillanceUnited States, 1981-2008. MMWR Morb Mortal Wkly Rep 2011;60: 689-93. 21637182
6 Schwarcz S, Weinstock H, Louie B et al. Characteristics of persons with recently acquired HIV infection: application of the serologic testing algorithm for recent HIV seroconversion in 10 US cities. J Acquir Immune Defic Syndr 2007;44: 112-5. doi:10.1097/01. qai.0000247228.30128.dc 17075386

7 Zetola NM, Kaplan B, Dowling T et al. Prevalence and correlates of unknown HIV infection among patients seeking care in a public hospital emergency department. Public Health Rep 2008;123(Suppl 3): 41-50. 19166088

8 Deblonde J, Meulemans H, Callens S, Luchters S, Temmerman M, Hamers FF. HIV testing in Europe: mapping policies. Health Policy 2011;103: 101-10. doi:10.1016/j.healthpol.2011.06.012 21794943

9 British HIV Association, British Association of Sexual Health and HIV, British Infection Society. UK National Guidelines for HIV Testing 2008. British HIV Association, 2008.

10 World Health Organization Regional Office for Europe. Scaling up HIV testing and counseling in the WHO European Region as an essential component of efforts to achieve universal access to HIV prevention, treatment, care and support.WHO, 2010.

11 European Centre for Disease Prevention and Control. HIV testing: increasing uptake and effectiveness in the European Union. ECDC, 2010.

12 Hamill M, Burgoine K, Farrell F et al. Time to move towards opt-out testing for HIV in the UK. BM/ 2007:334:1352-4. doi:10.1136/ bmj.39218.404201.9417600024

13 Berg LJ, Delgado MK, Ginde AA, Montoy JC, Bendavid E, Camargo CA Jr. Characteristics of U.S. emergency departments that offer routine human immunodeficiency virus screening. Acad Emerg Med 2012;19: 894-900. doi:10.1111/j.1553-2712.2012.01401.x 22849642

14 Hoover JB, Tao G, Heffelfinger JD. Monitoring HIV testing at visits to emergency departments in the United States: very-low rate of HIV testing. J Acquir Immune Defic Syndr 2013;62: 90-4. doi:10.1097/ QAl.0b013e3182742933 23018376

15 Choi J, Laibson D, Madrian B, Metrick A. Optimal defaults. Am Econ Rev 2003;93: 180-5doi:10.1257/000282803321947010.

16 Choi J, Laibson D, Madrian B, Metrick A. Saving for retirement on the path of least resistance. In: McCaffrey EJ, Slemrod J., eds. Behavioral Public Finance: Toward a New Agenda. Russell Sage Foundation, 2006: 304-51.

17 Simpson WM, Johnstone FD, Goldberg DJ, Gormley SM, Hart GJ. Antenatal HIV testing: assessment of a routine voluntary approach. BMJ 1999;318: 1660-1. doi:10.1136/bmj.318.7199.166010373168

18 Stringer EM, Stringer JS, Cliver SP, Goldenberg RL, Goepfert AR. Evaluation of a new testing policy for human immunodeficiency virus to improve screening rates. Obstet Gynecol 2001;98: 1104-8. doi:10.1016/S0029-7844(01)01631-311755561

19 Jayaraman GC, Preiksaitis JK, Larke B. Mandatory reporting of HIV infection and opt-out prenatal screening for HIV infection: effect on testing rates. CMAJ 2003;168: 679-82.12642422

20 White DAE, Scribner AN, Vahidnia F et al. HIV screening in an urban emergency department: comparison of screening using an opt-in versus an opt-out approach. Ann Emerg Med 2011;58(Suppl 1): S89-95. doi:10.1016/j.annemergmed.2011.03.032 21684416

21 White DAE, Sadoun T, Tran T, Alter HJ. Increased acceptance rates of HIV screening using opt-out consent methods in an urban emergency department. J Acquir Immune Defic Syndr 2011;58: 277-82. doi:10.1097/QAI.0b013e318231916d 21876449

22 Kendrick SR, Kroc KA, Couture E, Weinstein RA. Comparison of point-of-care rapid HIV testing in three clinical venues. AIDS 2004;18: 2208-10. doi:10.1097/00002030-200411050-00017 15577658

23 Calderon Y, Leider J, Hailpern S et al. High-volume rapid HIV testing in an urban emergency department. AIDS Patient Care STDS 2009;23: 749-55. doi:10.1089/apc.2008.0270 19698029

24 Lubelchek RJ, Kroc KA, Levine DL, Beavis KG, Roberts RR. Routine, rapid HIV testing of medicine service admissions in the emergency department. Ann Emerg Med 2011;58(Suppl 1): S65-70. doi:10.1016/j.annemergmed.2011.03.027 21684411

25 Lyons MS, Lindsell CJ, Ruffner AH et al. Randomized comparison of universal and targeted HIV screening in the emergency department. I Acquir Immune Defic Syndr 2013;64: 315-23. doi:10.1097/ QAl.0b013e3182a2161123846569

26 Lubelchek RJ, Kroc KA, Levine DL, Beavis KG, Roberts RR. Routine, rapid HIV testing of medicine service admissions in the emergency department. Ann Emerg Med 2011;58(Suppl 1): S65-70. doi:10.1016/j.annemergmed.2011.03.027 21684411

27 Haukoos JS, Hopkins E, Conroy AA et al. Denver Emergency Department HIV Opt-Out Study Group. Routine opt-out rapid HIV screening and detection of HIV infection in emergency department patients. JAMA 2010;304: 284-92. doi:10.1001/jama.2010.953 20639562

28 Rayment M, Thornton A, Mandalia S et al. HINTS Study Group. HIV testing in non-traditional settings--the HINTS study: a multi-centre observational study of feasibility and acceptability. PLoS One 2012;7: e39530. doi:10.1371/journal.pone.0039530 22745777 
29 Burns F, Edwards SG, Woods I et al. Acceptability and feasibility of universal offer of rapid point of care testing for HIV in an acute admissions unit: results of the RAPID project. PLoS One 2012;7: e35212. doi:10.1371/journal.pone.0035212 22558129

30 Merchant RC, Waxman MJ. HIV screening in health care settings: some progress, even more questions. JAMA 2010;304: 348-9. doi:10.1001/ jama.2010.996 20639572

31 White DAE, Scribner AN, Vahidnia F et al. HIV screening in an urban emergency department: comparison of screening using an opt-in versus an opt-out approach. Ann Emerg Med 2011;58(Suppl 1): S89-95. doi:10.1016/j.annemergmed.2011.03.032 21684416

32 Haukoos JS, Mehta SD, Harvey L, Calderon Y, Rothman RE. Research priorities for human immunodeficiency virus and sexually transmitted infections surveillance, screening, and intervention in emergency departments: consensus-based recommendations. Acad Emerg Med 2009;16: 1096-102. doi:10.1111/j.1553-2712.2009.00546.x 20053228

33 Mounier-Jack S, Nielsen S, Coker RJ. HIV testing strategies across European countries. HIV Med 2008;9(Suppl 2): 13-9. doi:10.1111/ j.1468-1293.2008.00585.x 18557864

$34 \mathrm{Ai} \mathrm{C}$, Norton EC. Interaction terms in logit and probit models. Econ Lett 2003;80: 123-9doi:10.1016/S0165-1765(03)00032-6.

35 Haukoos JS, Lyons MS, Lindsell CJ et al. Derivation and validation of the Denver Human Immunodeficiency Virus (HIV) risk score for targeted HIV screening. Am J Epidemiol 2012;175: 838-46. doi:10.1093/aje/kwr389 22431561

36 Haukoos JS, Hopkins E, Bucossi MM et al. Denver Emergency Department HIV Research Consortium. Brief report: Validation of a quantitative HIV risk prediction tool using a national HIV testing cohort. J Acquir Immune Defic Syndr 2015;68: 599-603. doi:10.1097/ QAl.0000000000000518 25585300

37 Haukoos JS, Hopkins E, Bender B et al. Denver Emergency Department HIV Testing Research Consortium. Use of kiosks and patient understanding of opt-out and opt-in consent for routine rapid human immunodeficiency virus screening in the emergency department. Acad Emerg Med 2012;19: 287-93. doi:10.1111/j.15532712.2012.01290.x 22435861

38 Merchant RC, Baird JR, Liu T, Taylor LE, Montague BT, Nirenberg TD. Brief intervention to increase emergency department uptake of combined rapid human immunodeficiency virus and hepatitis $C$ screening among a drug misusing population. Acad Emerg Med 2014:21: 752-67. doi:10.1111/acem.12419 25125271

39 Aronson ID, Marsch LA, Rajan S, Koken J, Bania TC. Computer-based video to increase HIV testing among emergency department patients who decline. AlDS Behav 2015;19: 516-22. doi:10.1007/s10461-0140853-5 25064047

40 Merchant RC, Seage GR, Mayer KH, Clark MA, DeGruttola VG, Becker BM. Emergency department patient acceptance of opt-in, universal, rapid HIV screening. Public Health Rep 2008;123(Suppl 3): 27-40. 19172704
41 Pisculli ML, Reichmann WM, Losina E et al. Factors associated with refusal of rapid HIV testing in an emergency department. AIDS Behav 2011:15: 734-42. doi:10.1007/s10461-010-9837-2 20978834

42 Merchant RC, Freelove SM, Langan TJ 4th et al. The relationship of reported HIV risk and history of HIV testing among emergency department patients. Postgrad Med 2010;122: 61-74. doi:10.3810/ pgm.2010.01.2100 20107290

43 Zetola NM, Grijalva CG, Gertler S et al. Simplifying consent for HIV testing is associated with an increase in HIV testing and case detection in highest risk groups, San Francisco January 2003-June 2007. PLoS One 2008;3: e2591. doi:10.1371/journal.pone.0002591 18596933

44 Kreps D, Porteus E. Temporal resolution of uncertainty and dynamic choice theory. Econometrica 1978;46: 185-200doi:10.2307/1913656.

45 Caplin A, Leahy J. Psychological expected utility theory and anticipatory feelings. Q J Econ 2001;116: 55-79doi:10.1162/0033553 01556347.

46 Koszegi B. Health anxiety and patient behavior. J Health Econ 2003;22: 1073-84. doi:10.1016/j.jhealeco.2003.06.00214604561

47 Mackellar DA, Hou SI, Whalen CC et al. WHBS Study Group. Reasons for not HIV testing, testing intentions, and potential use of an over-the-counter rapid HIV test in an internet sample of men who have sex with men who have never tested for HIV. Sex Transm Dis 2011:38: 419-28. doi:10.1097/OLO.0b013e31820369dd 21183863

48 Oster E, Shoulson I, Dorsey ER. Optimal expectations and limited medical testing: evidence from Huntington disease. National Bureau of Economic Research, 2011. (NBER Working Paper No 17629.)

49 Madrian BC, Shea DF. The power of suggestion: Inertia in $401(\mathrm{k})$ participation and savings behavior. Q J Econ 2001;116: 1149-87doi:10.1162/003355301753265543.

50 Consedine NS, Magai C, Krivoshekova YS, Ryzewicz L, Neugut Al. Fear, anxiety, worry, and breast cancer screening behavior: a critical review. Cancer Epidemiol Biomarkers Prev 2004;13: 501-10.15066912

51 Lerman C, Hughes C, Trock B et al. Genetic testing in families with hereditary nonpolyposis colon cancer. JAMA 1999;281: 1618-22. doi:10.1001/jama.281.17.161810235155

52 Lyons MS, Lindsell Cl, Haukoos IS et al. National Emergency Department HIV Testing Consortium. Nomenclature and definitions for emergency department human immunodeficiency virus (HIV) testing: report from the 2007 conference of the National Emergency Department HIV Testing Consortium. Acad Emerg Med 2009·16: 168-77. doi:10.1111/j.1553-2712.2008.00300.x 19076107

53 Stacey D, Bennett CL, Barry MJ et al. Decision aids for people facing health treatment or screening decisions. Cochrane Database Syst Rev 2011;10: CD001431. 21975733

C BMJ Publishing Group Ltd 2016

\section{Appendix}

